The Be Phenomenon in Early-Type Stars, IAU Colloquium 175

ASP Conference Series, Vol. 214, 2000

M. A. Smith, H. F. Henrichs, and J. Fabregat, eds.

\title{
Mid-term Photometric Periodic Variations in Be Stars HR 1960 and HR 2968
}

\author{
F. Carrier and G. Burki \\ Observatoire de Genève, Ch. des Maillettes 51, CH-1290 Sauverny, \\ Switzerland
}

\begin{abstract}
The Be stars HR 1960 and HR 2968 have been monitored from 1978 to 1998 in GENEVA photometry and from November 1989 to March 1993 by the HIPPARCos satellite. A spectroscopic survey of these stars started in September 1998. Both stars show luminosity variations with a period of $395 \mathrm{~d}$ and $371 \mathrm{~d}$ for HR 1960 and HR 2968 respectively.
\end{abstract}

\section{Introduction}

The homogeneity and accuracy of the photometric data obtained in the GENEVA system allow the detection of long-period variables, in particular among $\mathrm{Be}$ stars. In this paper, the photometric and spectroscopic variability of HR 1960 (HD 37935, HIP 26368) and HR 2968 (HD 61925, NGC 2451-187, HIP 37345) are described. These are two late B-type stars classified respectively $\mathrm{B} 9.5 \mathrm{Ve}$ and B6IVe in SIMBAD (Centre de Données Astronomiques de Strasbourg, CDS). Both stars were chosen initially as standard stars in the GENEVA photometric system. HR 1960 was used as a comparison star to analyse the variability of SN 1987A (Burki et al., 1991).

\section{Observations}

From 1978 to 1998 , respectively 676 and 358 measurements has been obtained on HR 1960 and HR 2968 in the GENEVA photometric system (Golay, 1980) with the photoelectric photometer P7 (Burnet \& Rufener, 1979) installed on the $40 \mathrm{~cm}$ and $70 \mathrm{~cm}$ Swiss telescopes in La Silla (ESO, Chile). In addition, 182 (HR 1960) and 147 (HR 2968) photometric measurements have been obtained by the HIPPARCOS satellite (ESA, 1997) from November 1989 to March 1993. To compare the magnitude $H p$ from HIPPARCOS with $V$, the relation of Carrier et al. (1999) has been used. In addition, spectroscopic observations were obtained from September 1998 with the CORALIE spectrograph installed on the $120 \mathrm{~cm}$ New Swiss telescope in La Silla.

\section{Physical parameters}

The physical parameters of HR 1960 can be derived from the photometric multicolour measurements and from the parallax determined by the HIPPARCOS satellite (ESA, 1997). By using the calibrations of Cramer (1999) for B-type 
Table 1. Physical parameters for HR 2968 and HR 1960.

\begin{tabular}{l|ccccccc}
\hline & $\log T_{\text {eff }}$ & $\begin{array}{c}\log g \\
{[\mathrm{cgs}]}\end{array}$ & $\begin{array}{c}E[B-V] \\
{[\mathrm{mag}]}\end{array}$ & $\begin{array}{c}d \\
{[\mathrm{pc}]}\end{array}$ & $\begin{array}{c}M_{V_{0}} \\
{[\mathrm{mag}]}\end{array}$ & $\begin{array}{c}M \\
{\left[M_{\odot}\right]}\end{array}$ & $\begin{array}{c}\log t \\
{[y r]}\end{array}$ \\
\hline HR 1960 & $4.02 \pm 0.02$ & $3.8 \pm 0.2$ & $\mathbf{0 . 0 2 1}$ & $262_{-28}^{+37}$ & $-0.89_{-29}^{+25}$ & $\mathbf{3 . 0}$ & $\mathbf{8 . 4 5}$ \\
HR 2968 & $4.09 \pm 0.02$ & $3.8 \pm 0.1$ & 0.010 & $\mathbf{3 5 8 \pm 2 2}$ & $-1.76 \pm 0.27$ & $\mathbf{5 . 9} \pm 0.5$ & $\mathbf{7 . 7 \pm 0 . 1}$ \\
\hline
\end{tabular}

stars, the values of Table 1 have been derived (Burki, 1999). In the case of HR 2968, the physical parameters are very accurately determined because the star is member of the cluster NGC 2451 B (Carrier et al., 1999).

\section{Photometric variations}

\subsection{HR 2968}
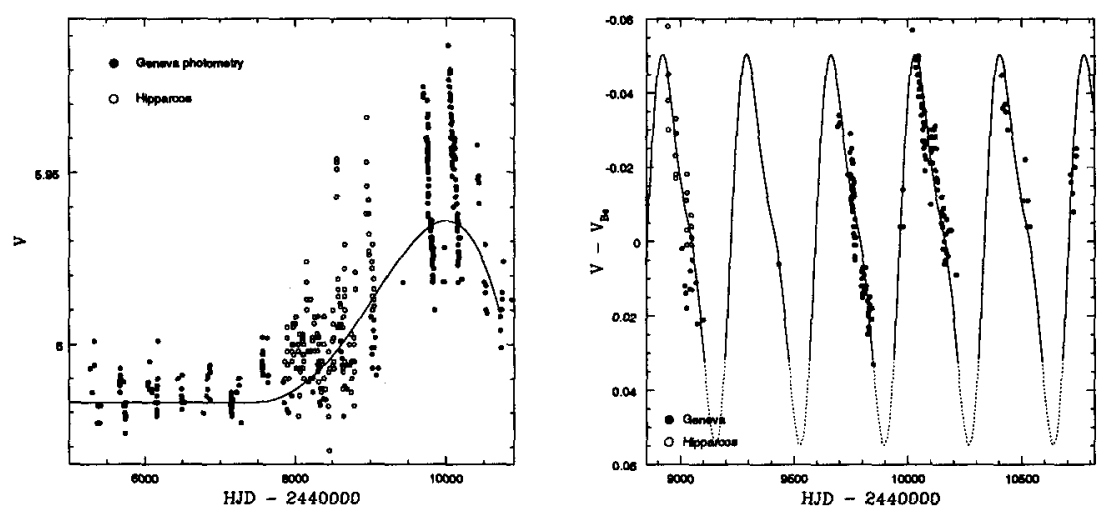

Figure 1. (Left) V magnitude measurements of HR 2968. The mean luminosity curve is drawn (long-term variability). Corrected $H p$ and $V$ magnitudes are identified by open and filled dots respectively. (Right) The mid-term variation. The period of the light curve is $371 \mathrm{~d}$.

HR 2968 shows exceptional luminosity variations (Fig. 1): i) The mean luminosity, which has been stable since 1978 (normal B-star phase), increased from 1990 to 1995 (Be phase), and then decreased until 1998; ii) In 1990 started also a periodic light variation with a period of $371 \mathrm{~d}$. The amplitude of the $V$ magnitude variation is enhanced during the long-term brightening and then seems to have been constant during five periods. GENEVA and HIPPARCOS magnitudes are in perfect agreement. The peak-to-peak value is about 0.10 mag in $V$.

\subsection{HR 1960}

HR 1960 exhibited nearly the same behaviour : i) The mean luminosity in $V$ has slightly increased during the survey; ii) A periodic light variation with a period of $395 \mathrm{~d}$ has been observed. The Discrete Fourier Transform of the GenEVA 

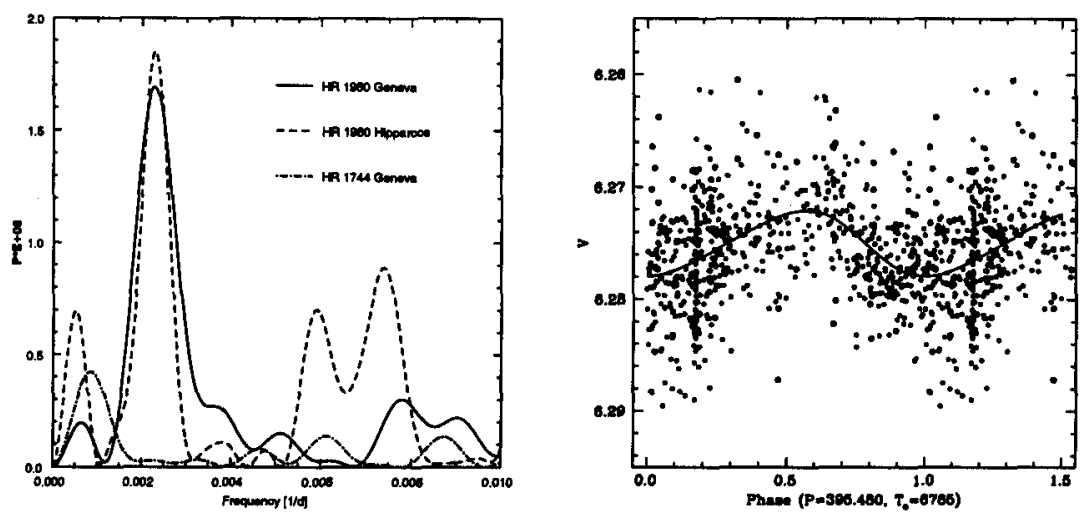

Figure 2. (Left) DFT of the $V$ magnitude measurements of HR 1960 and HR 1744. (Right) The mid-term $(\mathrm{P}=395.480 \mathrm{~d})$ light curve of HR 1960. Data from GENEVA photometry are identified by filled dots and HIPPARCOS $V$ magnitudes by open dots.

photometric data of HR 1960 clearly shows a peak at frequency $\sim 0.0023 \mathrm{~d}^{-1}$ (Fig. 2). DFT of the HIPPARCos data also presents its highest peak at the same frequency. During the monitoring of HR 1960, another comparison star was also intensively measured, HR 1744 . The DFT of the GENEVA photometric data of HR 1744 does not show any significant peak. Thus, the conclusion is that a mid-term periodic photometric variation was observed in HR 1960 with an extremely small semi-amplitude of $3 \mathrm{mmag}$.

5. On the origin of the variations and the spectroscopic survey
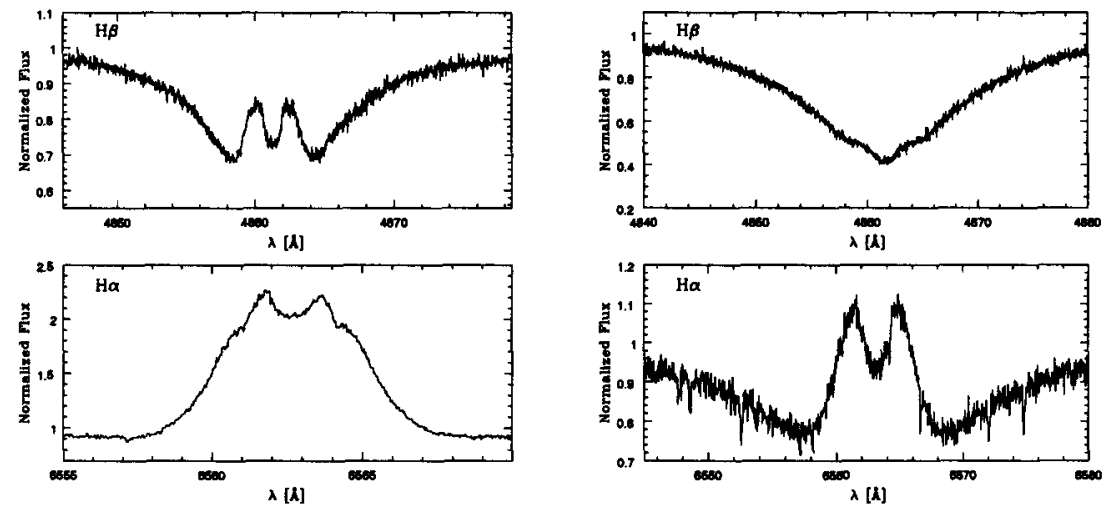

Figure 3. $\mathrm{H} \alpha$ and $\mathrm{H} \beta$ lines for HR 2968 (left) and HR 1960 (right). 

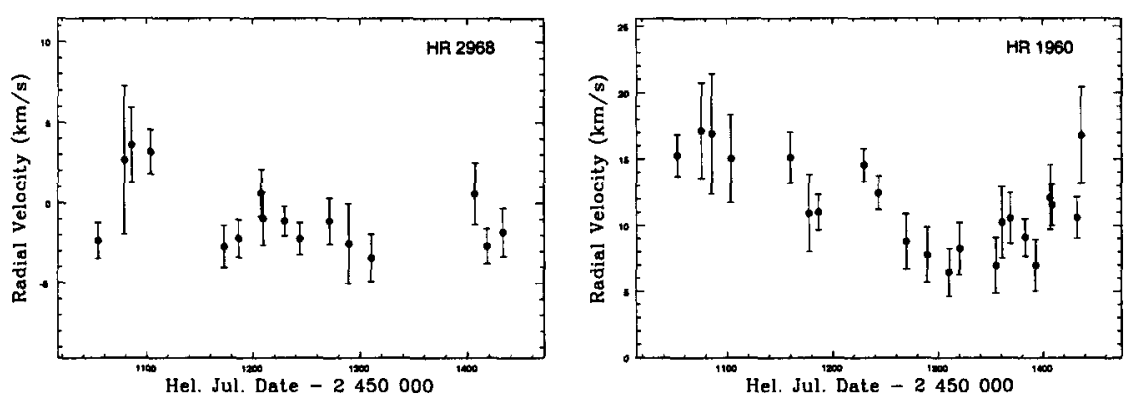

Figure 4. Radial velocity survey for HR 2968 and HR 1960.

A model is proposed to explain these periodic variabilities: the Be star is the main component of a binary system having an eccentric orbit. At each periastron passage, the companion star interacts gravitationally and/or radiatively with the disk around the Be star. This interaction induces a periodic modulation of the Iuminosity of the system. A crucial element is of course to know whether these Be stars are members of binary systems or not. Thus, a spectroscopic survey was started in 1998. Both stars are obviously in a Be-phase (see Fig. 3). The radial velocity variations are presented in Fig. 4. After only one year of survey, the results are especially encouraging in the case of HR 1960, since the variations seem in agreement with an orbital period equal to that one of the photometric variations (395 d). The case of HR 2968 is actually less clear. However, given the expected eccentricity and amplitude, an unambigous detection of the binarity character will need a monitoring during several orbital periods.

\section{Conclusion}

HR 2968 and HR 1960 are two Be stars which exhibit nearly the same photometric behaviour: both stars exhibit mid-term variations with periods of $371 \mathrm{~d}$ and $395 \mathrm{~d}$ for HR 2968 and HR 1960 respectively. According to the model proposed, each star would be the main component of a binary system. In the case of HR 1960, this model is supported by our radial velocity measurements, after only one year of survey.

\section{References}

Burnet M., Rufener F. 1979, A\&A 74, 54

Burki G., Cramer N., Nicolet B. 1991, A\&AS 87, 163

Burki G. 1999, A\&A 346, 134

Carrier F., Burki G., Richard C. 1999, A\&A 341, 469

Cramer N. 1999, New Astronomy Reviews, in press

ESA 1997, The HIPPARcos and Tycho Catalogues, ESA SP-1200

Golay M. 1980, Vistas in Astronomy 24, 141 